\title{
AN AUTOMATED HR DIAGRAM FOR NGC 6809 (M 55)
}

\author{
Michael J. Irwin \\ Institute of Astronomy, Cambridge \\ Virginia Trimble \\ University of Maryland and University of California
}

ABSTRACT: For decades, star counts and HR diagrams extending below the main sequence turnoff in globular clusters meant the work of Sandage (1957). The advent of large CCD's at the foci of large telescopes has changed this (McClure et al. 1985, Harris \& Hesser 1985, Christian \& Heasley 1986, Heasley et al. 1986, Penny \& Dickens 1986, Richer \& Fah1man 1986, Smith et al. 1986) and made clear that clusters differ in the shapes of their luminosity functions and in the morphology of their HR diagrams. We return here to photographic methods, which can capture an order of magnitude more images and so possibly reveal new details.

$\mathrm{J}$ and $\mathrm{R}$ Anglo-Australian Observatory plates of the rich, open southern globular cluster M55 have been scanned and analyzed with the Automated Place Measurement facility of the Inst. of Astronomy and a new crowdedfield algorithm (Irwin 1985). Almost 30,000 images were identifiable in both colors, of which slightly less than half represent cluster stars, extending nearly $3^{\mathrm{m}}$ below the main sequence turnoff. Comparison of images in the two colors confirms earlier conclusions (Irwin \& Trimble 1984) that (a) the luminosity function begins to flatten below $M=+5.5$ and (b) the radial profile, while generally well fit by a king model (with core and tidal radii of 2.2 and $26.6 \mathrm{pc}$ at $\mathrm{d}=4.8 \mathrm{kpc}$ ), shows several bumps and wiggles, of marginal statistical significance, that repeat from plate to plate.

The HR diagram (Fig. 1 is the raw data for the inner $16^{\prime}$ or $22 \mathrm{pc}$ radius) shows the usual features. The distribution of cluster stars around the ridge line of the main sequence is, at least, consistent with a sufficient population of close binaries to agree with a W UMa identification for the main sequence variables found in the earlier work. We have not yet attempted to fit evolutionary tracks or isochrones to the data, but the exceedingly sharp turnoff and a possible ridge-line job may prove interesting. 


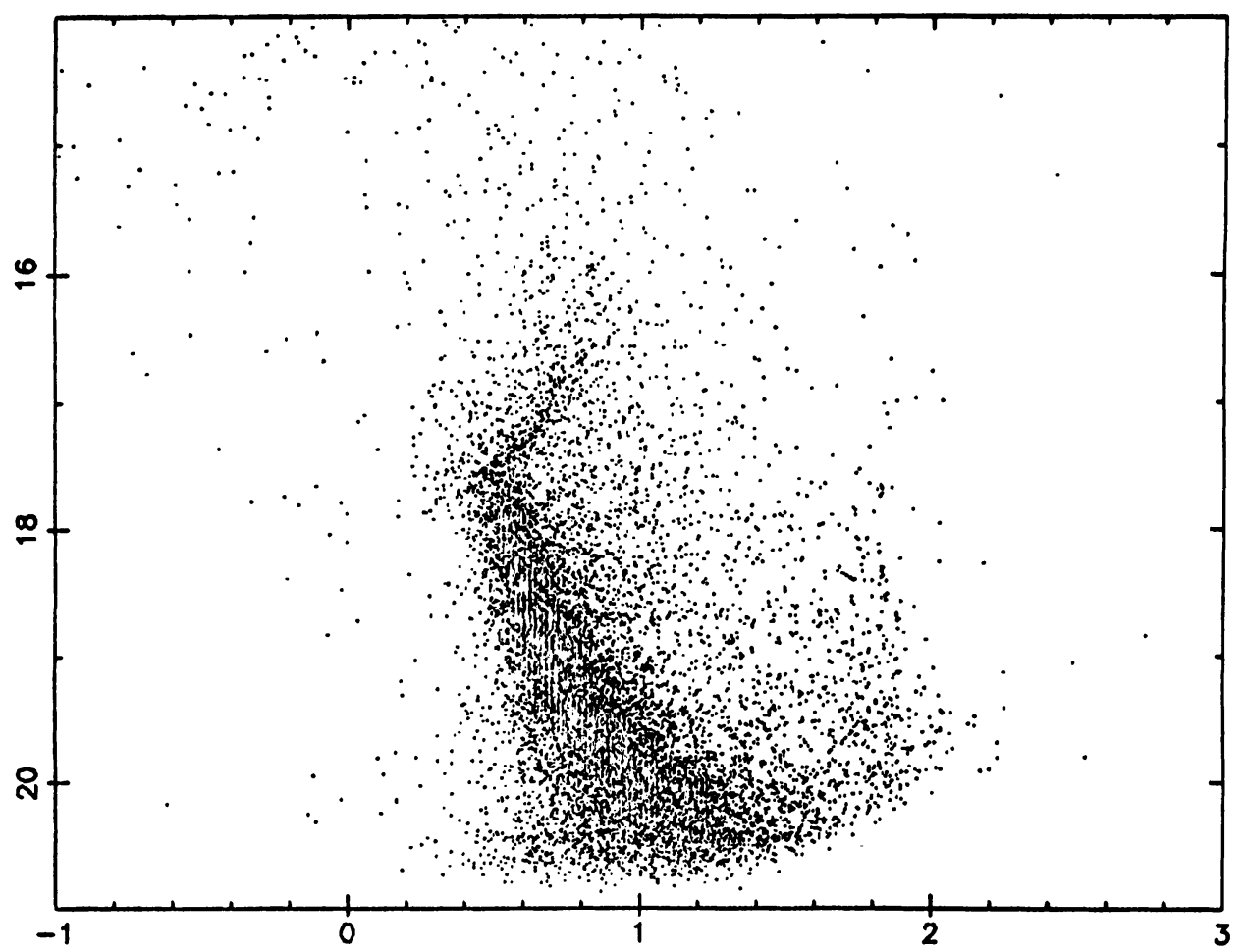

Fig.1. Automated HR diagram showing about 10,000 stars out to $r=16^{\prime}$ in the southern half of the cluster field. Axes are apparent magnitude $R$ and color $\mathrm{J}-\mathrm{R}$. Diagonal bottom cutoff is the plate limit $\mathrm{J}=22$ or $\mathrm{M}_{\mathrm{J}}$ about 8.3 at the apparent distance modulus, 13.7, of M 55 .

\section{REFERENCES}

Christian, C. A. and Heasley, J. N. 1986 Astrophys. J. 303, 216. Harris, W. and Hesser, J. 1985 in IAU Symposium No. 113, Dynamics of Star Clusters, J. Goodman and P. Hut, eds., Reide1, Dordrecht, p. 81 .

Heasley, J. N. Janes, K. A. and Christian, C. A. 1986 Astron. J. $91,1108$.

Irwin, M. J. 1985 Monthly Notices Roy. Astron. Soc. 214, 575/

Irwin, M. J. and Trimble, V. 1984 Astron. J. 89, 83.

McClure, R. D., Hesser, J. E., Stetson, P. B. and Stryker, L. L. 1985 Publ. Astron. Soc. Pacific 97, 665.

Penny, A. J. and Dickens, R. J. 1986 Monthly Notices Roy. Astron. Soc. 220,845 .

Richer, H. B. and Fahlman, G. G. 1986 Astrophys. J. 304, 273.

Sandage, A. R. 1957 Astrophys. J. 125, 422.

Smith, G. H., McClure, R. D., Stetson, P. B., Hesser, J. E. and Be1l, R. A. 1986 Astron. J. 91, 842 . 